\title{
Epidemiology of paediatric and adolescent fractures admitted to a South African provincial hospital
}

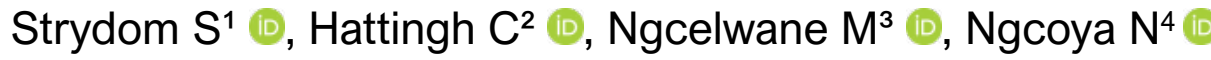 \\ $1 \mathrm{MBChB}$ (Pret); Orthopaedic Registrar, Department of Orthopaedics, University of Pretoria, Kalafong Tertiary Hospital, Pretoria, South Africa \\ 2 MBChB(Pret); Department of Orthopaedics, University of Pretoria, Steve Biko Academic Hospital, Pretoria, South Africa \\ 3 FCS(SA)Orth, MSc Orth(London); Department of Orthopaedics, University of Pretoria, Steve Biko Academic Hospital and Department of \\ Orthopaedics, Faculty of Health Sciences, University of Pretoria, Pretoria, South Africa \\ 4 FCS(SA)Orth; Department of Orthopaedics, University of Pretoria, Tembisa Provincial Tertiary Hospital, Tembisa, South Africa
}

Corresponding author: Dr Sven Strydom, Postnet Suite 169, Private Bag X8, Elarduspark, 0047; tel: +27 82778 9475; email: svenstrydom@gmail.com

\begin{abstract}
Background: There are limited studies available that examine the epidemiology of children and adolescents admitted with orthopaedic injuries in developing countries. Several unique factors in South Africa, such as a large socio-economic divide and a public health sector with limited resources, are suggested to influence injury patterns and fracture management. The data emanating from this study may play a role in identifying potential preventative measures. The aims of the study were to determine the age, sex, duration of admission, aetiology and management regimen of paediatric and adolescent patients admitted with fractures in a South African provincial hospital.
\end{abstract}

Patients and methods: We did a cross-sectional review of medical records for the period 1 January 2016 to 31 December 2017 at a South African provincial hospital. Convenience sampling was done, and demographic and clinical data was collected from patient records. All patients younger than 18 years at the time of injury who were admitted with fractures of the limbs, pelvis or spine were included. Patients with incomplete clinical records were excluded.

Results: A total of 731 patients were admitted during this period. After excluding patients with incomplete records, 526 (72\%) were included in the study. From these records we found a higher percentage of males admitted $(73 \%)$ and the average age was 7.72 years. The average duration of admission was 8.59 days. A fall on ground level was the most common mechanism of injury (70.0\%), followed by pedestrian vehicle accidents (12.2\%). The most frequently fractured regions were the forearm (36.4\%), humerus (26.5\%) and femur (18.9\%). Multiple fractures were sustained by $2.4 \%$ and additional non-skeletal injuries were sustained by $2.9 \%$. There was one fatality: a patient who presented with gunshot trauma.

Conclusion: Despite fall-related injuries being the most common cause for admission, pedestrian vehicle accident prevention can have the greatest effect on admissions. They require significantly longer hospitalisation and present more frequently with additional injuries. By implementing safety strategies and improving road infrastructure, we can theoretically decrease the number of serious paediatric admissions.

Level of evidence: Level 4

Keywords: epidemiology, paediatric, adolescent, fracture, admission, management

Citation: Strydom S, Hattingh C, Ngcelwane M, Ngcoya N. Epidemiology of paediatric and adolescent fractures admitted to a South African provincial hospital. SA Orthop J 2020;19(2):92-96. http://dx.doi.org/10.17159/2309-8309/2020/v19n2a6

Editor: Dr Greg Firth, Royal London Hospital, London, England

Received: October 2019

Accepted: January 2019

Published: May 2020

Copyright: ( 2020 Strydom S. This is an open-access article distributed under the terms of the Creative Commons Attribution Licence, which permits unrestricted use, distribution and reproduction in any medium, provided the original author and source are credited.

Funding: No funding was received for this study.

Conflict of interest: The authors declare they have no conflicts of interest that are directly or indirectly related to the research. 


\section{Introduction}

Trauma remains a leading cause of death and disability in children worldwide. ${ }^{1}$ The management of fractures in low-income countries is often sub-optimal due to resource limitations, predisposing patients to an increased risk of physical disability. ${ }^{2}$ South Africa has an injury-related mortality rate that is six times greater than the global average, with the number of road traffic accident injuries being double the global average. ${ }^{3}$

Fractures during childhood are common, with studies showing incidence ranging from $25 \%$ to $34 \% .^{2,4}$ Other studies found boys had an increased risk of sustaining a fracture.5,6 Paediatric fractures strongly relate to lifestyle and environment, making risk assessment and incidence studies vital to monitor trends and plan for the future. ${ }^{7}$ In developing countries like South Africa most parents are required to work, but cannot afford or do not have access to safe day-care facilities, therefore children are often left unsupervised. ${ }^{8}$ The incidence of fractures requiring admission appears to be on the rise; one study reported an increase of $38 \%$ over a nine-year period. ${ }^{7}$ Hospital care remains the most expensive component of any healthcare system. ${ }^{9}$ Galano et al. ${ }^{1}$ highlighted that understanding the patterns of traumatic injuries is vital in establishing effective injury prevention programmes and adjusting treatment protocols to optimise patient outcome. Thus, we may be able to better weigh the cost of prevention against the cost of treatment to optimise resource management.

The majority of studies investigating the epidemiology of fractures in a local community are from American and Eurasian countries. There are currently no South African studies nor are there any trauma databases in Gauteng on injury surveillance to facilitate predictions on trends in fracture patterns. This makes it difficult for local government to identify high risk areas where safety programmes need to be instituted to prevent childhood injuries or provide guidance for resource planning and allocation.

\section{Patients and methods}

We did a cross-sectional review of medical records at Tembisa Provincial Tertiary Hospital, a South African provincial hospital which services an estimated population of 1.2 to 1.4 million in Gauteng. All children and adolescents aged less than 18 years at the time of injury and admitted with a fracture of the limbs, pelvis or spine were included in our study. We did convenience sampling of the period 1 January 2016 to 31 December 2017. Standard demographic data was analysed. The mechanism of injury was taken from the history given on presentation and the fractures sustained were grouped according to the region of the body involved. Data on the admission duration, definitive treatment of the fracture received, and additional non-orthopaedic injuries sustained were collected from the clinical notes. Incomplete files were excluded from the study. Data analysis was done on the software suite SAS v9.4 and descriptive statistics were used to describe the data.

\section{Results}

During the two-year study period, 731 patients were admitted with fractures meeting our criteria. A total of 526 (72\%) had complete files and were included in the study. We noted a high male-tofemale ratio, with $73 \%$ of fractures occurring in males. The mean (standard deviation - SD) age at the time of injury was 7.7 (4.5) years; with an interquartile range (IQR) of 4 to 11 years. The mean age for males to sustain a fracture was 8.3 years, while females had a mean age of 6.1 years. Admissions according to age group are demonstrated in Figure 1.

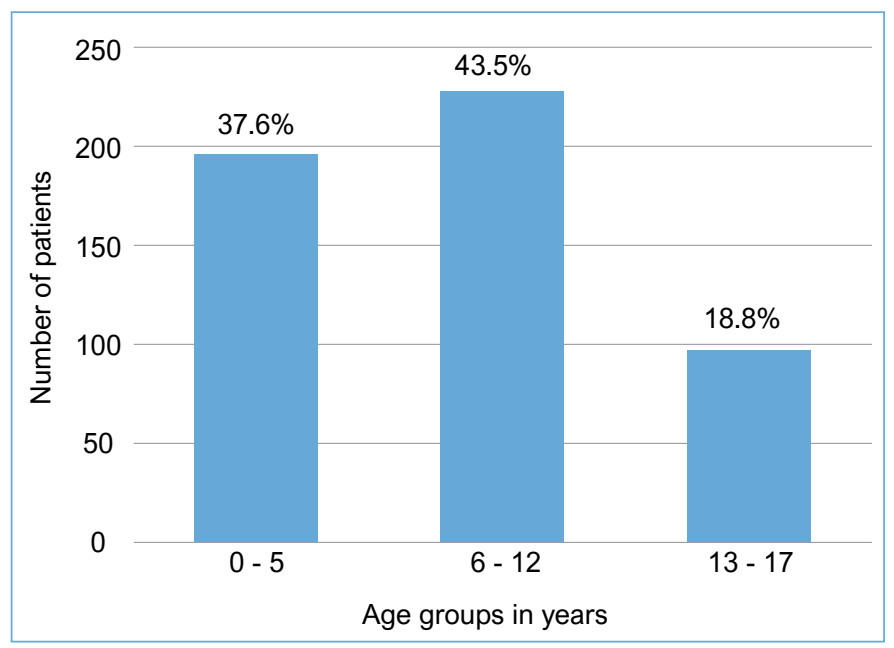

Figure 1. Admissions per age group

The mean duration of admission was 8.6 days, with an IQR of 3 to 10 days. Figure 2 demonstrates the admission duration trend. Patients with multiple fractures remained in hospital for a mean of 13.5 days. Those admitted with femur fractures were admitted for the longest duration on average (18.8 days), followed by pelvis fractures (13.0 days) and tibia/fibula fractures (8.8 days). The mechanisms of injury resulting in the longest average duration of admission were railroad injuries (27.0 days) and pedestrian vehicle accidents (PVAs) (14.7 days). Patients with additional nonorthopaedic injuries were admitted on average for 22.3 days.

A breakdown of the admissions based on the five commonest mechanisms of injury is provided in Table I. Of those injured in PVAs, $76.6 \%$ were aged 12 years or younger. Less common mechanisms were motor vehicle accidents (1.7\%), gunshot and railroad injuries (both $0.4 \%$ ). There were no confirmed or suspected non-accidental causes of injury, defined as deliberate injury caused by a parent/ caregiver. A total of 539 fractures were diagnosed, with $13(2.4 \%)$ children sustaining fractures to multiple regions and six $(1.1 \%)$ children having multiple admissions during the study period for both refractures and new fractures involving different bones. The most common region fractured was the forearm, accounting for $36.4 \%$ of the fractures. This was followed by the humerus $(26.5 \%)$, femur $(18.9 \%)$, leg $(12.2 \%)$, pelvis and foot (both 1.9\%), hand $(1.1 \%)$, spine $(0.6 \%)$ and clavicle and scapula $(0.6 \%)$. Additional non-musculoskeletal injuries were sustained in $15(2.9 \%)$ of the admissions. With the exception of one patient, these were all due to high energy trauma. There was one mortality: a patient who sustained gunshot injuries resulting in fractures of his forearm and spine and abdominal trauma.

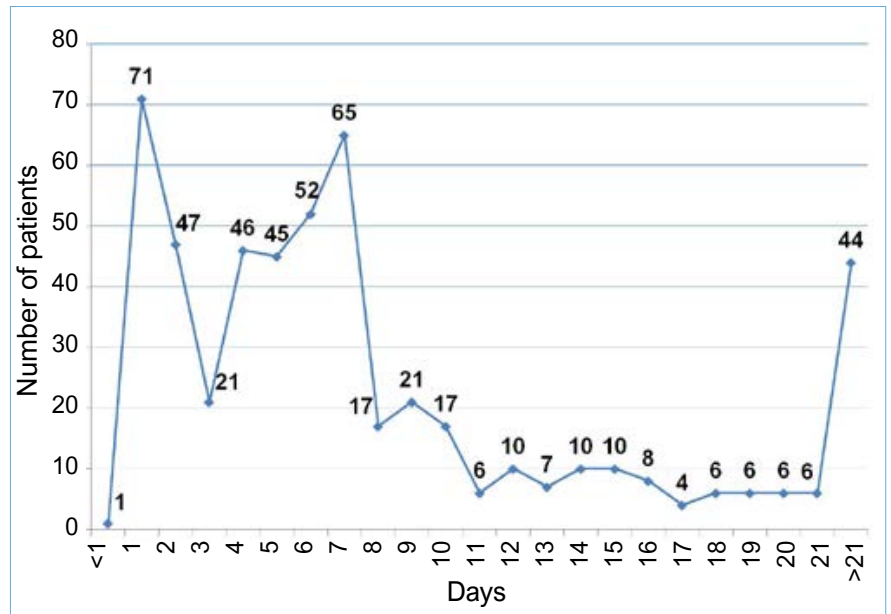

Figure 2. Admission duration trend 
Table I: Admission breakdown based on the commonest mechanisms of injury

\begin{tabular}{|c|c|c|c|c|c|c|c|c|c|}
\hline \multirow{2}{*}{$\begin{array}{l}\text { Mechanism } \\
\begin{array}{l}\text { Fall } \\
\text { (ground level) }\end{array}\end{array}$} & \multirow{2}{*}{$\begin{array}{l}\mathbf{n}(\%) \\
368 \\
(70)\end{array}$} & \multirow{2}{*}{$\begin{array}{l}\text { M:F n } \\
(\%) \\
\\
276: 92 \\
(75: 25)\end{array}$} & \multirow{2}{*}{$\begin{array}{c}\begin{array}{c}\text { Mean } \\
\text { age } \\
\text { (years) }\end{array} \\
7.6\end{array}$} & \multirow{2}{*}{$\begin{array}{c}\begin{array}{c}\text { Mean } \\
\text { admission } \\
\text { duration } \\
\text { (days) }\end{array} \\
7.3\end{array}$} & \multicolumn{2}{|c|}{ Fracture n (\%) } & \multirow{2}{*}{$\begin{array}{c}\begin{array}{c}\text { Additional } \\
\text { injuries } \\
\mathrm{n}(\%)^{\mathrm{b}}\end{array} \\
1(7)\end{array}$} & \multicolumn{2}{|c|}{ Management n (\%) } \\
\hline & & & & & Hand & $2(<1)$ & & Bed rest & $1(<1)$ \\
\hline & & & & & Forearm & $172(44)$ & & Sling & $2(1)$ \\
\hline & & & & & Humerus & $117(30)$ & & Traction & $35(10)$ \\
\hline & & & & & Clavicle/scapula & $1(<1)$ & & Plaster only & $94(26)$ \\
\hline & & & & & Spine & $1(<1)$ & & Manipulation and plaster & $121(33)$ \\
\hline & & & & & Pelvis & $1(<1)$ & & Manipulation and K-wire & $81(22)$ \\
\hline & & & & & Femur & $56(14)$ & & ORIF (plate and screws) & $23(6)$ \\
\hline & & & & & Leg & $21(5)$ & & Intramedullary nail & $3(1)$ \\
\hline & & & & & Foot & $21(5)$ & & Transfer to private & $7(2)$ \\
\hline & & & & & & & & Absconded & $1(<1)$ \\
\hline & & & & & Multiple fractures ${ }^{a}$ & $4(31)$ & & & \\
\hline \multirow{12}{*}{$\begin{array}{l}\text { Pedestrian } \\
\text { vehicle } \\
\text { accident }\end{array}$} & $\begin{array}{c}64 \\
(12)\end{array}$ & $\begin{array}{l}41: 23 \\
(64: 36)\end{array}$ & 8.4 & 14.7 & Forearm & $4(6)$ & $10(67)$ & Bed rest & $3(5)$ \\
\hline & & & & & Humerus & $1(2)$ & & Sling & $2(3)$ \\
\hline & & & & & Clavicle/scapula & $2(3)$ & & Traction & $7(11)$ \\
\hline & & & & & Pelvis & $5(7)$ & & Plaster only & $21(32)$ \\
\hline & & & & & Femur & $19(28)$ & & Manipulation and plaster & $12(18)$ \\
\hline & & & & & Leg & $33(49)$ & & Manipulation and K-wire & $5(8)$ \\
\hline & & & & & Foot & $4(6)$ & & $\begin{array}{l}\text { Soft tissue procedure } \\
\text { and plaster }\end{array}$ & $1(2)$ \\
\hline & & & & & & & & ORIF (plate and screws) & $5(8)$ \\
\hline & & & & & Multiple fractures ${ }^{a}$ & $4(31)$ & & Intramedullary nail & $6(9)$ \\
\hline & & & & & & & & External fixator & $1(2)$ \\
\hline & & & & & & & & Primary amputation & $1(2)$ \\
\hline & & & & & & & & Transfer to private & $2(3)$ \\
\hline \multirow[t]{7}{*}{ Fall $(>1 \mathrm{~m})$} & $\begin{array}{l}35 \\
(7)\end{array}$ & $\begin{array}{c}26: 9 \\
(74: 26)\end{array}$ & 6.4 & 8.0 & Forearm & $11(31)$ & 0 & Traction & $2(6)$ \\
\hline & & & & & Humerus & $17(49)$ & & Plaster only & $5(14)$ \\
\hline & & & & & Spine & $1(3)$ & & Manipulation and plaster & $15(43)$ \\
\hline & & & & & Femur & $4(11)$ & & Manipulation and K-wire & $10(29)$ \\
\hline & & & & & Leg & $2(6)$ & & ORIF (plate and screws) & $2(6)$ \\
\hline & & & & & & & & Transfer to private & $1(3)$ \\
\hline & & & & & Multiple fractures ${ }^{a}$ & 0 & & & \\
\hline \multirow[t]{8}{*}{ Crush injury } & $\begin{array}{l}32 \\
(6)\end{array}$ & $\begin{array}{l}21: 11 \\
(66: 34)\end{array}$ & 6.7 & 11.4 & Hand & $3(9)$ & 0 & Bed rest & $2(6)$ \\
\hline & & & & & Forearm & $4(11)$ & & Traction & $11(34)$ \\
\hline & & & & & Humerus & $4(11)$ & & Plaster only & $11(34)$ \\
\hline & & & & & Pelvis & $3(9)$ & & Manipulation and plaster & $3(9)$ \\
\hline & & & & & Femur & $12(34)$ & & Manipulation and K-wire & $1(3)$ \\
\hline & & & & & Leg & $6(17)$ & & $\begin{array}{l}\text { Soft tissue procedure } \\
\text { and plaster }\end{array}$ & $1(3)$ \\
\hline & & & & & Foot & $3(9)$ & & Intramedullary nail & $3(9)$ \\
\hline & & & & & Multiple fractures ${ }^{a}$ & $3(23)$ & & & \\
\hline \multirow[t]{5}{*}{ Assault } & $\begin{array}{l}10 \\
(2)\end{array}$ & $\begin{array}{c}9: 1 \\
(90: 10)\end{array}$ & 10.1 & 5.9 & Hand & $1(10)$ & 0 & Plaster only & $4(44)$ \\
\hline & & & & & Humerus & $3(30)$ & & Manipulation and plaster & $2(22)$ \\
\hline & & & & & Femur & $2(20)$ & & Manipulation and K-wire & $2(22)$ \\
\hline & & & & & Leg & $4(40)$ & & ORIF (plate and screws) & $1(11)$ \\
\hline & & & & & Multiple fractures ${ }^{a}$ & 0 & & & \\
\hline
\end{tabular}




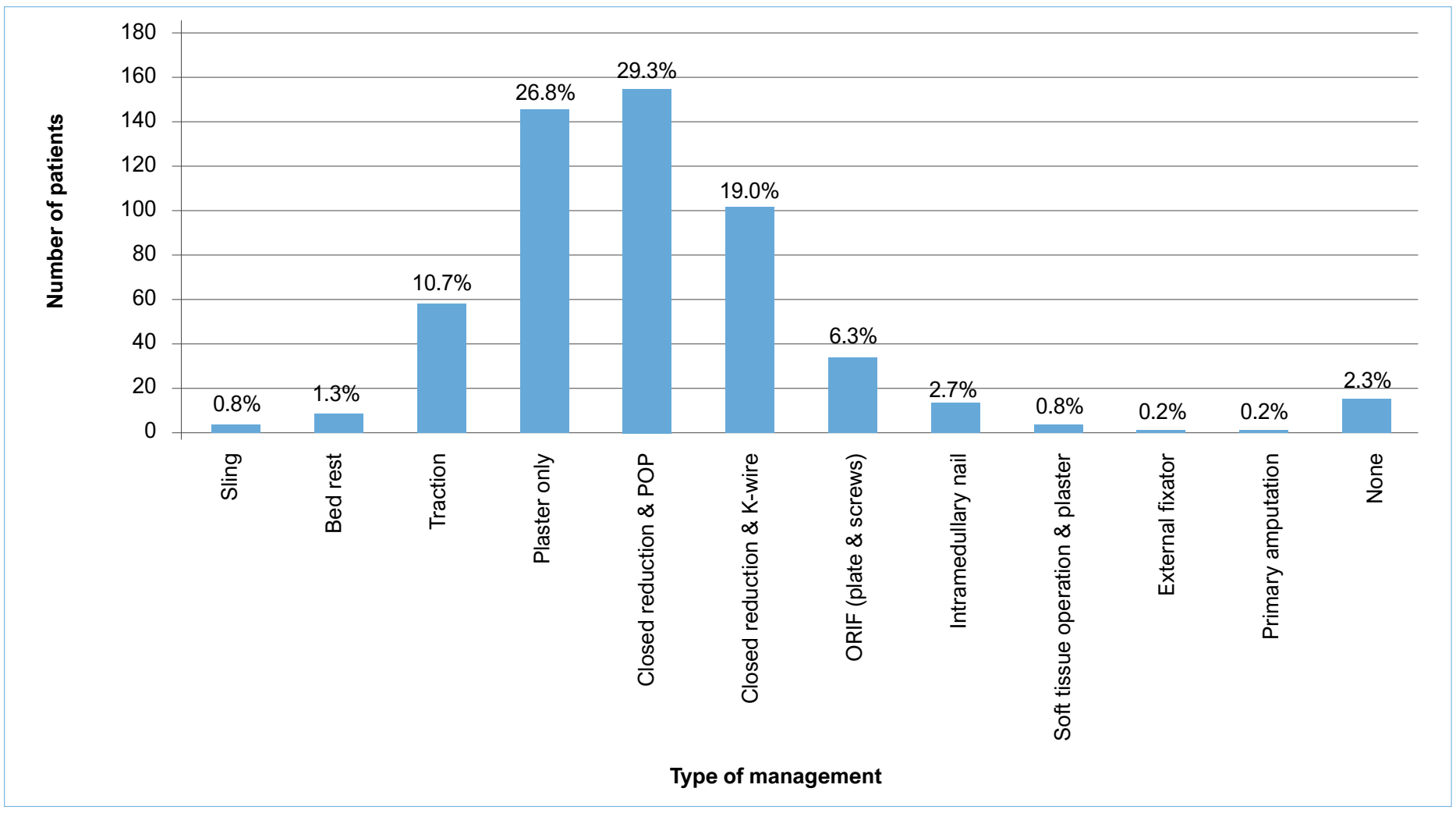

Figure 3. Summary of definitive management received

Our research shows the following results related to the 539 fractures: $57.5 \%$ received definitive management in theatre and $39.9 \%$ received conservative management in the ward. Definitive treatment with plaster was the most frequently used method of treatment $(56.1 \%)$, while hardware was used in $28.2 \%$ of patients taken to theatre. Figure 3 provides a breakdown of the management received. Patients who received plaster only were admitted due to transport difficulties at night or for monitoring of perfusion due to swelling. Those who received closed reduction were managed in theatre under general anaesthesia.

\section{Discussion}

There is limited data on the number of children and adolescents admitted with fractures in developing countries like South Africa. While there are numerous international studies, their findings have limited application to our population due to different socio-economic circumstances, an increased trauma burden and a unique disease profile that strain a healthcare system with limited resources.

Even though we found a higher male-to-female ratio admitted with fractures, the mean age for admission with a fracture was in keeping with previous publications. The greatest number of admissions occurred among the 5- to 6-year-old age groups. This highlights that children at this age have developed independent mobility and explorative behaviour, yet still lack sufficient cognitive awareness of environmental hazards and avoidance skills. ${ }^{8,10}$

Similar to other publications, we also found a fall on ground level to be the most common mechanism of injury. ${ }^{2,8}$ Approximately one out of 18 children with a fall-related fracture required admission in a Hong Kong-based study. ${ }^{9}$ What is distressing is that PVAs were the second most common (12.2\%) cause of injury which results in admission, with the majority occurring in children aged 12 years or younger. We found that these patients presented more frequently with lower limb fractures, accounting for $50 \%$ of tibia/fibula fractures and $18.9 \%$ of all the femur fractures admitted. Hitosugi et al. ${ }^{10}$ noted that patients injured in a PVA are more likely to require admission as they tend to suffer more severe injuries. This was confirmed in our study with a large number of the patients with multiple fractures and $66.7 \%$ of additional non-skeletal injuries being present in the group injured in PVAs. They were also admitted for 6.1 days longer than the average. Most children involved in PVAs are from lowerincome communities, where a major risk factor is the absence of usable sidewalks, ${ }^{11}$ which is the norm in communities such as the one under discussion. The incidence of PVAs has been found to increase with age, ${ }^{7}$ with the majority occurring in children walking to school. ${ }^{8}$ This emphasises the need for environmental modification in high risk areas, such as adequate speed breakers, sidewalks in local communities and safe public transport to schools. ${ }^{8}$ Several studies have also recommended educational programmes directed at both children and parents, ${ }^{8,10-12}$ while others have shown a significant reduction in road traffic-related injuries after implementation of such prevention strategies. ${ }^{5,13}$ It is evident that this is one area where we can have a great impact on the number of children admitted with fractures, thus reducing the associated morbidity and strain on healthcare resources.

Clinicians should always maintain a high index of suspicion for non-accidental injuries. The incidence of confirmed non-accidental injuries ranges from $1 \%$ to $7.7 \%$ in children presenting with musculoskeletal injuries, $8,14,15$ with the highest incidence among children less than 2 years of age. ${ }^{14}$ Risk factors for child abuse include multiple fractures in different stages of healing, femur fractures in children less than 1 year old, unemployed parents, unplanned pregnancies and children less than 3 years old. ${ }^{15}$ In a low-income community such as ours, a number of these risk factors may be present. Clinicians can miss the subtle signs of non-accidental injuries, especially in public hospitals where patient volumes can often overwhelm the limited number of healthcare workers. In our study, 12 of the 13 children aged less than 1 year were admitted with femur fractures. Also, none of the injuries caused by physical assault were suspected to be abuse-related. 
Since none of the patients were suspected of being victims of nonaccidental injuries, this may indicate that we are not recognising these victims. We can thus recommend that all healthcare workers be regularly re-educated on the signs of abuse; even easily visible educational posters may assist with identification of individuals at risk.

Femur fractures remain one of the fractures requiring a longer hospitalisation period due to the large number treated conservatively, ${ }^{1,2}$ as was also confirmed in our study. Reducing the duration of admission has a direct impact on the cost of care and Guifo et al. ${ }^{2}$ described the use of home traction to achieve this reduction in admission duration. This is however not practical in our setting, as parents often lack the funds necessary for frequent hospital visits, and staff shortages prevent home visits.

Our study is reliant on diligent note-keeping by treating healthcare providers and accurate data capturing by clerks. A total of $205(28 \%)$ patients were excluded due to incomplete records or missing files. Additionally, as there is no set protocol on which patients need admission; it is left to the discretion of the on-duty doctor. This could result in an inexperienced doctor admitting minor injuries, while a more experienced doctor would have treated a similar patient on an out-patient basis. Because of staff turnover, the average level of experience remains fairly constant through the years. Therefore, the results should still reflect the amount of resources required to adequately manage these patients annually. However, to better understand the epidemiology of paediatric and adolescent fractures, the number of patients managed on an outpatient basis should also be considered. Unfortunately, the records were insufficient to allow us to trace these patients' files to include them in our study. Further limitations include lack of information on specific locations of humerus fractures and how many open fractures were included in the cohort.

\section{Conclusion}

The need to provide safer environments for children has already been recognised in South Africa. However, trauma-related injuries remain a leading cause of death and disability in children, continuing to place a burden on hospitals. Despite a fall on ground level being the most common cause of admission, effective PVA prevention could have the greatest effect on trauma admissions. This is the second commonest mechanism of injury in our communities, with the majority occurring in young children. They require significantly longer hospitalisation and present more frequently with additional injuries. By implementing safety strategies and improving road infrastructure, we can theoretically decrease the number of serious paediatric admissions and so reduce the burden on our healthcare system. In an already resource-strained system, any reduction in patient load or unnecessary wastage of resources can improve quality of healthcare for all in the community.

\section{Ethics statement}

Prior to commencement of the study ethical approval was obtained from the following ethical review boards: University of Pretoria's Health Science Research Ethics Committee (ref: 357/2019) and the National Heath Research Database (ref: GP 201906 038).

This article does not contain any studies with humans or animals. For this study formal consent was not required.

\section{Declaration}

The authors declare authorship of this article and that they have followed sound scientific research practice. This research is original and does not transgress plagiarism policies.

\section{Author contributions}

SS contributed to the conceptualisation, design, data collection, data analysis and manuscript preparation.

$\mathrm{CH}$ contributed to data collection, data analysis and manuscript preparation.

$\mathrm{MN}$ contributed to the design and manuscript preparation.

$\mathrm{NN}$ contributed to the design and manuscript preparation.

\section{ORCID}

Strydom S (D) https://orcid.org/0000-0002-6288-4388 Hattingh C (D) https://orcid.org/0000-0002-8477-4657

Ngcelwane M (D) https://orcid.org/0000-0001-7564-3308

Ngcoya N (D) https://orcid.org/0000-0001-7122-0287

\section{References}

1. Galano G, Vitale M, Kessler M, Hyman J, Vitale M. The most frequent traumatic orthopaedic injuries from a national pediatric inpatient population. J Pediatr Orthop, 2005;25(1):39-44. http:// dx.doi.org/10.1097/00004694-200501000-00010.

2. Guifo M, Tochie J, Oumarou B, et al. Paediatric fractures in a sub-saharan tertiary care centre: A cohort analysis of demographic characteristics, clinical presentation, therapeutic patterns and outcomes. Pan Afr Med J. 2017;27:46. http://dx.doi. org/10.11604/pamj.2017.27.46.11485

3. Norman R. The high burden of injuries in South Africa. Bull World Health Organ. 2007;85(09):695-702. http://dx.doi.org/10.2471/ blt.06.037184.

4. Hedström E, Svensson O, Bergström U, Michno P. Epidemiology of fractures in children and adolescents. Acta Orthop. 2010;81(1):148-53. http://dx.doi.org/10.3109/17453671003628780.

5. Mäyränpää $M$, Mäkitie $O$, Kallio $P$. Decreasing incidence and changing pattern of childhood fractures: A population-based study. J Bone Miner Res. 2010;25(12):2752-59. http://dx.doi.org/10.1002/ jbmr.155.

6. Ferrari S, Chevalley T, Bonjour J, Rizzoli R. Childhood fractures are associated with decreased bone mass gain during puberty: An early marker of persistent bone fragility? J Bone Miner Res. 2005;21(4):501-507. http://dx.doi.org/10.1359/jbmr.051215.

7. Issin A, Kockara N, Oner A, Sahin V. Epidemiologic properties of pediatric fractures in a metropolitan area of turkey. Medicine. 2015;94(43):e1877. http://dx.doi.org/10.1097/ MD.0000000000001877.

8. Hussain S, Dar T, Beigh A, et al. Pattern and epidemiology of pediatric musculoskeletal injuries in Kashmir valley, a retrospective single-center study of 1467 patients. J Pediatr Orthop B. 2015;24(3):230-37. http://dx.doi.org/10.1097/ BPB.0000000000000146.

9. Lee J, Tung K, Li T, et al. Fall-related attendance and associated hospitalisation of children and adolescents in Hong Kong: A 12-year retrospective study. BMJ Open. 2017;7(2):e013724. http:// dx.doi.org/10.1136/bmjopen-2016-013724.

10. Hitosugi M, Kawato H, Gomei S, Mizuno K, Tokudome S. Severity of child pedestrian injuries due to bonnet-type-vehicle collision. Pediatr Int. 2013;55(5):624-28. http://dx.doi.org/10.1111/ ped.12140.

11. Calhoun A, McGwin G Jr, King W, Rousculp M. Pediatric pedestrian injuries: A community assessment using a hospital surveillance system. Acad Emerg Med. 1998;5(7):685-90. http:// dx.doi.org/10.1111/j.1553-2712.1998.tb02486.x.

12. Pasco J, Lane S, Brennan-Olsen S, et al. The epidemiology of incident fracture from cradle to senescence. Calcif Tissue Int. 2015;97(6):568-76. http://dx.doi.org/10.1007/s00223-015-0053-y.

13. Ferraz-Torres M, Belzunegui-Otano $T$, Martínez-García O, IriarteCerdán L, Salgado-Reguero E. Epidemiological characteristics and overall burden of accidental injuries in Navarra, Spain. $J$ Trauma Nurs. 2016;23(4):231-36. http://dx.doi.org/10.1097/ JTN.0000000000000222.

14. Servaes S, Brown S, Choudhary A, et al. The etiology and significance of fractures in infants and young children: a critical multidisciplinary review. Pediatr Radiol. 2016;46(5):591-600. http:// dx.doi.org/10.1007/s00247-016-3546-6.

15. Fong $\mathrm{C}$, Cheung $\mathrm{H}$, Lau $\mathrm{P}$. Fractures associated with non-accidental injury - an orthopaedic perspective in a local regional hospital. Hong Kong Med J. 2005;11(6):445-51. 